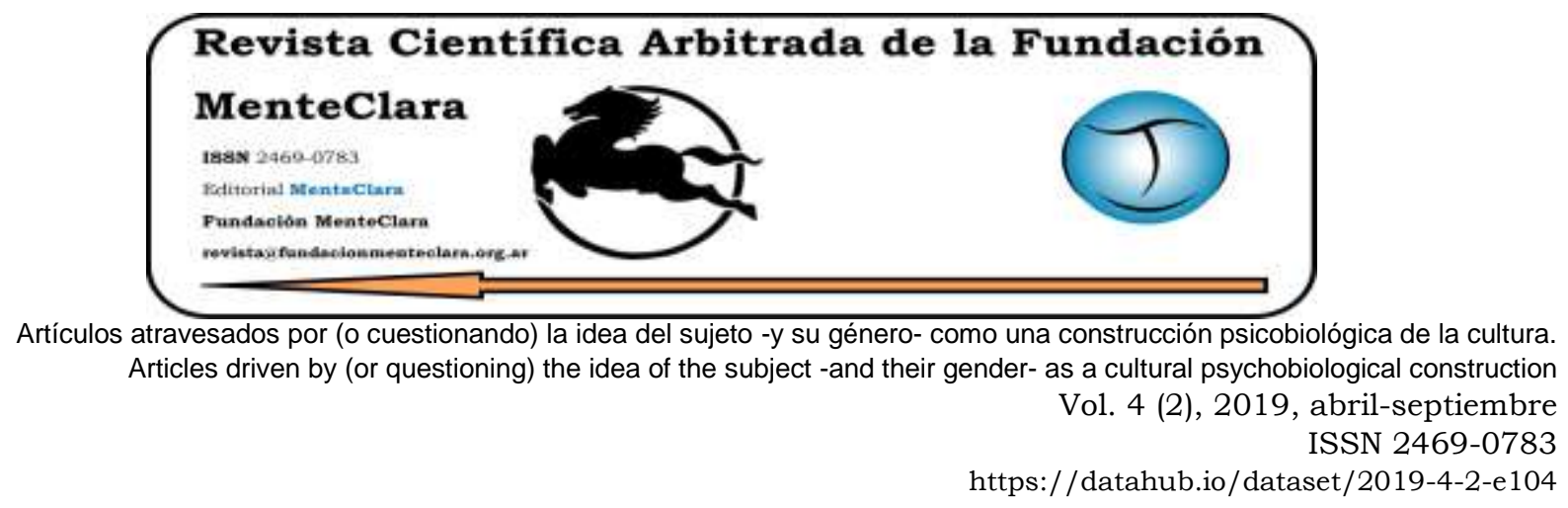

\title{
TANTRA Y LOS LÍMITTES DEL POTENCIAL FEMENINO EN LA RESPUESTA SEXUAL
}

TANTRA AND THE LIMIITS OF FEMALE POTENTİAL IN THE SEXUAL RESPONSE

H. Ümit Sayin humitsayin@gmail.com Institute of Forensic Sciences, İstanbul University

Cerrahpaşa Medical School, Cerrahpaşa, Aksaray-İstanbul

Cómo citar este artículo / Citation: Sayin, H. Ü. (2019). "Tantra y los límites del potencial femenino en la respuesta sexual". Revista Científica Arbitrada de la Fundación MenteClara, 4(2) abril-septiembre 2019, 203-243.

DOI: https://doi.org/10.32351/rca.v4.2.104

Copyright: (C) 2019 RCAFMC. Este artículo de acceso abierto es distribuido bajo los términos de la licencia Creative Commons Attribution 4.0 International License (CC BY 4.0). Recibido: 02/08/2019. Aceptado: 23/09/2019 Publicación online: 30/10/2019

Financiación: Esta investigación y revisión fue financiada por la Unidad de Coordinación de Proyectos de Investigación Científica (BAP) y la Universidad de Estambul.

Conflicto de intereses: Ninguno que declarar.

\section{Resumen}

Este artículo explica algunos de los nuevos hallazgos sobre la sexualidad femenina, la Respuesta Sexual Expandida (RSE) y los orgasmos prolongados-ampliados en comparación con las descripciones de las antiguas filosofias tántricas y taoístas. E1 orgasmo femenino y las "experiencias pico" femeninas tienen su adecuado reconocimiento en la antigua literatura de la India, China y Extremo Oriente. Al igual que los orgasmos tántricos, la respuesta sexual expandida se definió recientemente como: la capacidad de alcanzar orgasmos de larga duración y/o prolongados y/o múltiples y/o sostenidos y/o status orgasmus que dura más tiempo y es más intenso que los patrones de orgasmos clásicos definidos en la literatura occidental. Occidente empezó a comprender la verdadera naturaleza del orgasmo femenino en la segunda mitad del siglo XX con el uso de métodos de investigación científicos objetivos y 
racionales. En esta revisión se presentan descripciones detalladas de estos fenómenos en el marco de investigaciones clínicas actuales.

\begin{abstract}
This article explains explains some of the novel findings on female sexuality, ESR and prolonged-expanded orgasms, in comparison with the old Tantric and Taoist philosophies. The female orgasm and the female "peak experiences" have their proper recognition in the ancient literature of India, China and the Far East. Similar to Tantric Orgasms, ESR (Expanded Sexual Response) has been defined recently as: the capaciti able to attain long lasting and/or prolonged and/or multiple and/or sustained orgasms and/or status orgasmus that lasted longer and more intense than the classical orgasm patterns defined in the western literature. The West started to understand the real nature of female orgasm in the second half of twentieth century using objective and rational scientific investigation methods. This review presents detailed descriptions of these phenomena in the context of current clinical research.
\end{abstract}

Palabras Claves: Tantra; respuesta sexual expandida; RSE; orgasmo femenino; status orgasmus; orgasmo tántrico

Keywords: Tantra; expanded sexual response; ESR; female orgasm; status orgasmus; tantric orgasm 


\section{Introducción}

El orgasmo femenino y las "experiencias pico" -o, como la llamó Jung, experiencia numinosa- femeninas han sido bien documentadas en la literatura antigua de la India, China y Extremo Oriente (Vatsyayana, 1883), (Chang, 1977), (Chang, 1983), (Wu, 1996), (Schwartz, 1999), (Chia, 2002) (Chia, 2005), (Mumford, 2005), (Michaels, 2008).

Las culturas orientales habian tratado de descubrir los limites y la extensión de la respuesta orgásmica femenina durante siglos (Wu, 1996), (Chang, 1977, 1983) a diferencia de las culturas occidentales, donde, durante siglos, el placer y el orgasmo de las mujeres fueron entendidos como "pecado" y no se consideraban aceptables como en las culturas india tántrica o china taoísta.

El taoísmo en China, consideraba el cuerpo humano como una parte indispensable de la naturaleza existente o el "Tao". Esta visión holística de la filosofia humana condujo a la antigua medicina china a descubrir muchos otros detalles del cuerpo humano, así como la respuesta sexual, que era una función importante de la fisiología humana y que no se consideraba algo indecente.

Las culturas católicas, judia y protestante, así como las sociedades islámicas, durante siglos, percibieron el placer femenino como algo malo, malvado y satánico, mientras que el coito era solo un medio reproductivo. Las culturas del Lejano Oriente, consideraban el placer corporal del hombre y la mujer, como una función saludable y vital del cuerpo natural.

La fisiología sexual humana fue investigada por métodos científicos objetivos después de la revolución sexual durante la década de 1950 en occidente. El Kama Sutra, cuando fue traducido por Sir Richard Burton, fue inmediatamente prohibido en Inglaterra en el siglo XIX. Algunos 
científicos pioneros, como el Dr. Havelock Ellis, el Dr. Sigmund Freud, el Dr. Wilhelm Reich, el Dr. Alfred Kinsey, el Dr. William Masters y Virginia Johnson, el Dr. Beverly Whipple, el Dr. Barry Komisaruk tuvieron que desafiar las normas de la sociedad y dar grandes pasos e incluso saltos, antes de que la sexualidad humana, en occidente, comenzara a considerarse como una función normal y crucial de la fisiología humana.

Hoy en día, la fisiología sexual humana se investiga utilizando técnicas muy sofisticadas, como f (MRI) y técnicas modernas de fisiología y grabación. Los occidentales han vuelto a descubrir los puntos de vista y aspectos orientales de la fisiología sexual humana durante los últimos 60-70 años.

Hoy, el concepto "Tantra", en occidente tiene una mala reputación debido a muchos prejuicios sociales y culturales, mientras que el nombre de Tantra en la India y occidente ha sido utilizado y prostituido por muchos charlatanes, traficantes de sexo y monjes budistas vendedores de "curas" mágico-milagrosas". El concepto "Tantra" ha sido investigado por científicos y académicos, en términos científicos objetivos, desde muchas décadas; pero, aun así no se entiende correctamente y generalmente se malinterpreta.

El objetivo de este artículo de revisión es presentar algunos de los últimos hallazgos y conceptos sobre la sexualidad humana y el orgasmo femenino, como la respuesta sexual expandida-RSE- que tiene fuertes correlaciones históricas de las culturas tántricas del Lejano Oriente.

\footnotetext{
${ }^{1}$ En la siguiente investigación se puede verificar como algunos líderes del budismo mahayana, que de declaran seguidores del Dalai Lama, al igual que otros "gurúes" dicen: "Manjushri negro purifica contaminaciones, impurezas y cura enfermedades contagiosas como el ébola y el SIDA" (Gómez, 2017) https://fundacionmenteclara.org.ar/revista/index.php/RCA/article/view/11/71\#Apendice_1b
} 


\section{Formas tántricas y taoistas de amor}

En Tantra y también en la vía del amor taoísta, durante siglos, la prolongación del placer sexual de las mujeres se consideró un enfoque esencial. La antigua receta taoísta china para la sexualidad masculina también proponía a los varones prolongar las relaciones sexuales durante un par de horas, mientras que, según los curanderos chinos, perder el semen o la "energía yang" era un mal hábito.

También en los rituales tántricos, se alentó a los hombres y mujeres a prolongar la relación sexual, sin tener orgasmos -especialmente a los hombres-.

En ambas culturas orientales, se aconsejó a los hombres que no eyacularan antes de que las mujeres experimentaran muchos orgasmos múltiples (Vatsyayana, 1883), (Chang, 1977, 1983), (Wu, 1996), (Schwartz, 1999), (Chia 2002, 2005), (Mumford, 2005). Una tendencia similar existía en las antiguas culturas griegas, romanas y helenas; particularmente en los cultos dionisíacos. Durante los rituales dionisíacos, se alentó a prolongar la sexualidad y el orgasmo femenino (Sayin, 2014-b).

\section{La naturaleza de la respuesta sexual expandida del orgasmo femenino-RSE-}

El orgasmo femenino es una respuesta neuropsicológica y una experiencia máxima que resulta de la tensión sexual acumulada, la estimulación sexual, la excitación y la acumulación sexual interna, que se acompaña de una descarga neuronal y psicológica.

Como Masters \& Johnson, Hartman y Fithian investigaron en condiciones de laboratorio, la respuesta orgásmica femenina se complementa con la contracción de cierta musculatura voluntaria e 
involuntaria, como la vulva, la vagina, el útero, los músculos del piso pélvico (PFM), algunos de los músculos del cuerpo -pierna, abdomen, músculos pélvicos- (Masters \& Johnson, 1966), (Sayin 2010, 2012-a).

Algunos investigadores (Mah y Binik, 2001) han descrito el orgasmo femenino como:

- Reacción neurohormonal de los órganos del músculo liso y contracción de los homólogos de los músculos eyaculadores (Campell y Peterson, 1953).

- Contracciones vaginales espásticas que ocurren en los niveles de tensión más altos (Glenn y Kaplan, 1968).

- Respuesta sensorial-motora reflexiva que involucra contracciones genitopelvicas (Kaplan, 1974).

- Respuesta sensorial-motora reflexiva a la estimulación sexual (KlineGraber y Graber, 1975).

- Liberación de vaso concentración y miotonía por estimulación sexual (Masters y Johnson, 1966).

- Estados alterados de conciencia (Davidson y Davidson, 1980).

- Acción refleja involuntaria acompañada de contracciones uterinas / vaginales (Reubens, 1982).

- Fenómeno psíquico, una sensación -descarga neuronal cerebralprovocada por el efecto acumulativo en ciertas estructuras cerebrales de estímulos apropiados originados en las zonas erógenas periféricas (Alzate, 1985).

- Experiencias complejas de cambios genitales, cambios en el tono del músculo esquelético / movimientos semi voluntarios, cambios cardiovasculares / respiratorios (Bancroft, 1989). 
- Sensación repentina e intensa justo antes de las contracciones genitopelvicas (Hite, 1976).

- Acmé de placer sexual con convulsiones rítmicas del cuerpo de órganos perineales / reproductivos, cambios cardiovasculares y respiratorios, liberación de tensión sexual (Schiavi y Segraves, 1995).

- Potencia orgástica; capacidad de rendirse al flujo de energía biológica; capacidad para descargar la excitación sexual contenida a través de convulsiones involuntarias y placenteras del cuerpo (Reich, 1973).

Cuando observamos la naturaleza del orgasmo femenino, aunque existen patrones similares al orgasmo masculino, parece ser muy diferente a la eyaculación masculina dependiendo de la mujer que lo experimenta. En un orgasmo femenino único clásico, parece haber diferentes patrones que contribuyen a los cambios corporales (Sayin, 2012-h):

- Cambios en todo el cuerpo: taquicardia, presión arterial elevada, hiperventilación, sudoración, extensión de algunos grupos musculares -Ej., piernas y pies-, tensión muscular, enrojecimiento sexual, vasodilatación en las arteriolas cutáneas y aumento de golpes en la sangre venosa.

- Cambios genito-pélvicos: erección del complejo del clitoris y el glande del clítoris, agrandamiento del área del punto G y esponja uretral, lubricación, contracción involuntaria de la vagina, útero y cuello uterino, contracción voluntaria e involuntaria de los músculos del piso pélvico -músculos $\mathrm{PC}-$, contracción involuntaria del esfinter anal. 
- Cambios psicológicos: alivio de la tensión, sensación de descarga, disminución de la ansiedad, felicidad, euforia, relajación, satisfacción, sensación subjetiva de deshacerse de la tensión eléctrica y muscular, estados alterados de conciencia-ASC-.

Según lo medido por Masters \& Johnson, la duración de la contracción del área genito-pélvica ocurre a intervalos de 0.8 segundos (Masters \& Johnson, 1966).

Aunque los hombres tienen un período refractario después de un orgasmo, o eyaculación, para recuperar la erección, se ha documentado que las mujeres tienen la capacidad de continuar teniendo múltiples clímax si son estimuladas de manera continua y adecuada (Schwartz, 1999) (Bodansky, 2000) (Taylor, 2000, 2002), (Komisaruk, 2006), (Sayin 2010, 2012a, 2014, 2015).

Según lo descrito por Masters \& Johnson, algunas mujeres pueden alcanzar un estado orgásmico que puede durar 43 segundos, acuñado como status orgasmus (Masters \& Johnson, 1966).

En algunas mujeres que han desarrollado RSE, los orgasmos múltiples y el status orgasmus pueden variar en duración y en número de orgasmos menores contenidos en el patrón orgásmico largo.

Últimamente, estos orgasmos prolongados y los métodos para alcanzarlos se han publicado en muchos libros y artículos (Rhodes, 1991), (Schwartz, 1999), (Bodansky, 2000), (Taylor, 2000, 2002), (Komisaruk, 2006), (Sayin, 2010, 2011a-c, 2012a, 2014, 2015),

\section{(Deadone, 2011).Hemos definido el status orgasmus como:}

El status orgasmus es la forma continua de orgasmos combinados y/u orgasmos clitorales / vaginales que duran desde 1 minuto hasta 10-15 minutos o más. Durante el status orgasmus se experimenta 
un estado orgásmico continuo y se cree que muy pocas mujeres alcanzan el status orgasmus, por ejemplo, menos del 1\% de toda la población femenina.

El status orgasmus se puede ver en los orgasmos vaginales y del clitoris, sin embargo, se ve principalmente como una forma expandida / extendida de orgasmos combinados, en los que se desencadenan los reflejos del orgasmo vaginal y del clitoris al mismo tiempo. Estados orgásmicos similares y orgasmos de cuerpo completo también se definen en la literatura tántrica.

La duración puede variar de mujer a mujer. El status orgasmus fue definido por primera vez por Masters \& Johnson con una duración de 43 segundos en una mujer en 1966. Hoy se estima que el status orgasmus continúa durante 1 a 2 minutos, mientras que puede durar de 10 a 15 minutos, un estado orgásmico prolongado que termina con un orgasmo gigante -Big-O- que brinda un gran alivio y satisfacción al final.

En la mayoria de las experiencias de status orgasmus, generalmente hay un período refractario de 10 a 15 minutos. El número de orgasmos menores en un status orgasmus puede exceder de 5-10 a 20-30 -algunas mujeres afirman que esta cantidad ronda los 50-. En el status orgasmus se cree que la combinación de nervios pudentales, pélvicos, hipogástricos y vagales median el mecanismo desencadenante al mismo tiempo.

Como fenómeno novedoso, "los orgasmos de RSE y orgasmos múltiples" parecen ser diferentes en muchos aspectos de los orgasmos individuales clásicos, según lo definido por Masters \& Johnson y Kaplan (Ver Fig.1), (Masters \& Johnson, 1966), (Kaplan, 1981), (Rhodes, 1991), (Schwartz, 1999), (Taylor, 2000, 2002), (Deadone, 2011), (Sayin, 2010, 2011c, 2012a-bh, 2014, 2015): 
- La duración de los orgasmos individuales en el tren orgásmico puede aumentar.

- La duración de toda la experiencia orgásmica puede aumentar, como durar decenas de minutos.

- La intensidad de los orgasmos menores individuales generalmente aumenta junto con la longitud del tren orgásmico.

- El número de orgasmos menores en el tren orgásmico puede estar más allá del patrón orgásmico normal y promedio, como superar los 20-30 orgasmos en decenas de minutos.

- Se informa que el placer y el alivio sexual son mucho más comparados con uno o varios orgasmos múltiples.

- Sin un periodo refractario, un nuevo estado orgásmico comienza después de cada orgasmo, sin pasar a una fase de resolución, mientras que el estado de conciencia orgásmica se mantiene durante mucho tiempo -por ejemplo, de un par de minutos a decenas de minutos u horas-.

- Aunque puede haber algunas formas de estados alterados de conciencia -ASC- en algunos orgasmos individuales de algunas mujeres, la mayoría de los orgasmos de ESR y status orgasmus están acompañados de ASC, mientras que la percepción del tiempo, el continuo espacio-tiempo puede verse profundamente alterado. Se informaron 85 estados de ánimo diferentes en publicaciones anteriores (Sayin, 2011c, 2012-h, 2015).

- Según lo informado por muchas mujeres con RSE, los orgasmos parecen tener ansioliticos, antidepresivos, eufóricos, miorrelajantes, sedantes, analgésicos y "efectos alucinógenos de acción aguda y corta" (Sayin, 2011c), (Sayin, 2012a-dh, 2015). 
A

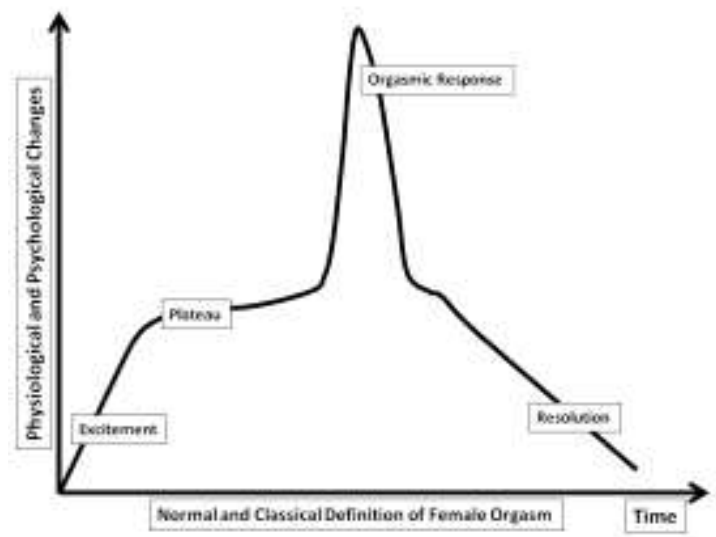

$\mathrm{C}$

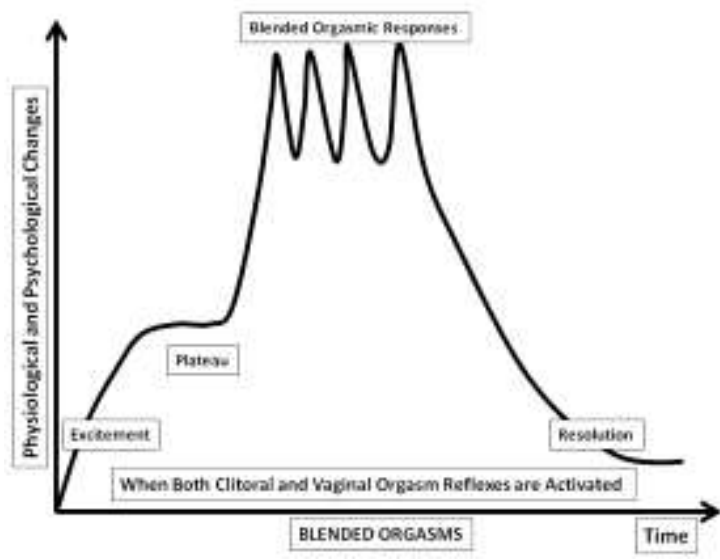

B

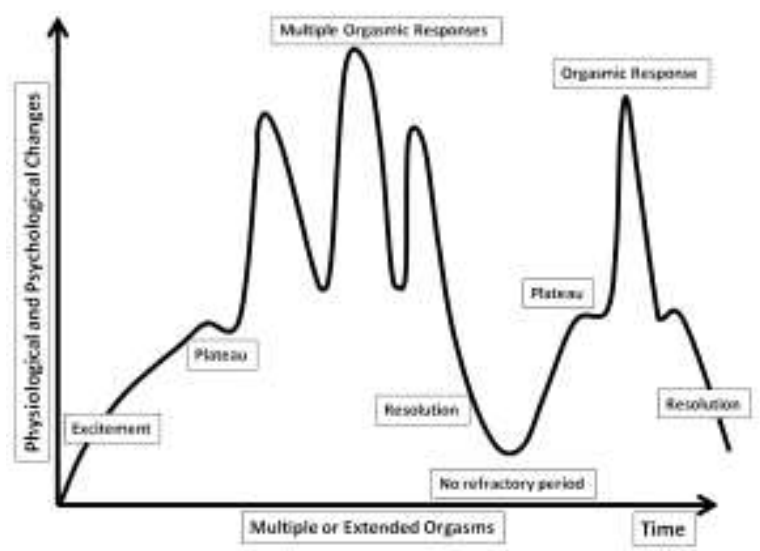

$\mathrm{D}$

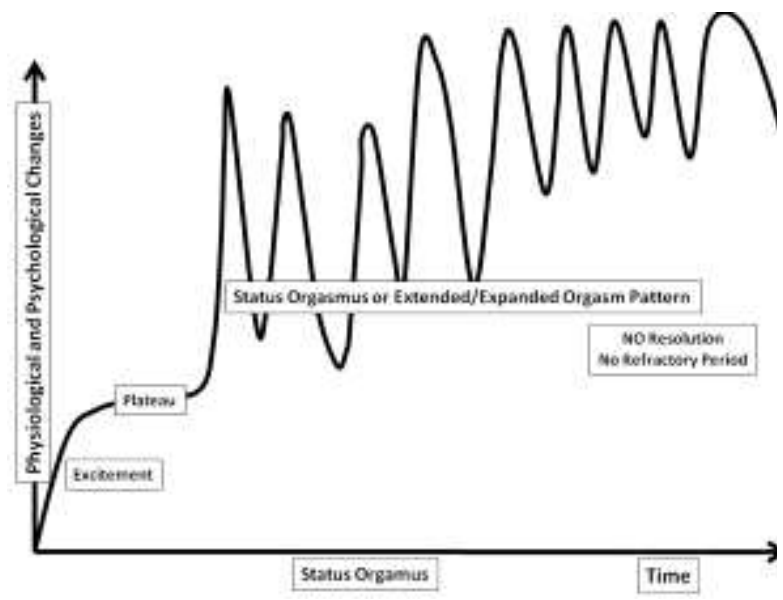

Figura 1:

A) Patrón de orgasmo femenino clásico, definido en la literatura médica clásica.

B) Patrón de orgasmo femenino múltiple.

C) Patrón de orgasmo femenino combinado.

D) Orgasmo expandido prolongado o status orgasmus. 


\section{Respuesta sexual expandida -RSE-}

La RSE es un fenómeno recientemente definido (Rhodes, 1991), (Taylor, 2000, 2002), (Armagan, 2012), (Sayin, $2011 \mathrm{a}-\mathrm{c}, 2012 \mathrm{a}-\mathrm{bh}, 2014$, 2015) de la siguiente manera:

"Ser capaz de alcanzar orgasmos duraderos $y$ / o prolongados y / o múltiples y / o sostenidos y / o un status orgasmus que duró más y más intenso que los patrones clásicos de orgasmos definidos en la literatura" (Sayin, 2011 ac, 2012-ah).

En la literatura oriental, china, india y tántrica, se han informado experiencias orgásmicas mejoradas similares en mujeres (Vatsyayana, 1883), (Chang 1977, 1983), (Schwartz, 1999), (Chia 2002, 2005), (Mumford, 2005), (Michaels 2008).

Recientemente, algunos estudios de mujeres orgásmicas también revelaron una forma de estados alterados de conciencia - ASC-durante los orgasmos (Komisaruk et al, 2006), (Sukel, 2011).

Hay un número creciente de informes de mujeres que experimentan una forma de ASC durante orgasmos prolongados y muy intensos, que forman el concepto novedoso de la RSE.

Sin embargo, en esos estudios no se realizó una clasificación de la respuesta sexual para abordar una pregunta tal que, si estas mujeres experimentaban un patrón de orgasmo mejorado y RSE, o no. La mayor parte del cuestionario que investigó los ASC durante los orgasmos se preparó para buscar un patrón de orgasmo promedio de mujer.

La hipótesis principal en los estudios de RSE fue: "La respuesta sexual, la conciencia orgásmica y el placer orgásmico se pueden mejorar, prolongar $y$ expandir en la mujen. Aunque una pequeña proporción de mujeres ha alcanzado o puede alcanzar la RSE hoy, la RSE es un fenómeno aprendido que puede desarrollarse en muchas mujeres mediante la 
capacitación y la educación, particularmente mediante la capacitación tántrica.

Para determinar los principales parámetros y mecanismos de RSE, hemos investigado las características principales de las mujeres que han desarrollado RSE (Taylor, 2000, 2002), (Armagan, 2012), (Sayin, 2011ab, 2012a-bch, 2014, 2015):

1) Las mujeres con RSE experimentaron orgasmos vaginales, clitorianos y mixtos, según lo descrito por Ladas et al. (Ladas, 1982), (Taylor, 2000, 2002), (Armagan, 2012), (Sayin 2010, 2011a-bc, 2012-abcdh, 2014, 2015).

2) Las mujeres con RSE experimentaron orgasmos múltiples en la mayoría de sus actividades sexuales (Schwartz, 1999), (Taylor, 2000, 2002), (Mumford, 2005), (Armagan, 2012), (Sayin 2010, $2011 \mathrm{a}-\mathrm{bc}$, 2012-abcd).

3) Las mujeres con RSE pudieron alcanzar orgasmos duraderos y / o prolongados y / o múltiples y / o sostenidos y / o status orgasmus que duraron más que el orgasmo único clásico y / o los patrones de orgasmo múltiple definidos en la literatura (Schwartz, 1999), (Taylor, 2000, 2002), (Sayin, 1993, 2010, 2011a-bc, 2012a-bcdh, 2014, 2015).

4) Las mujeres con RSE afirmaron tener fuertes músculos del piso pélvico (PFM) en comparación con las mujeres NRSE (Ladas, 1981), (Britten, 1983), (Sayin, 2010, 2011b, 2012a-bh).

5) La libido de las mujeres RSE fue muy alta en comparación con las mujeres NRSE (Armagan, 2012), (Sayin, 2012a-b).

6) Las mujeres con RSE describieron un fenómeno llamado orgasmos del punto G (Ladas, 1982), (Taylor, 2000, 2002), (Armagan, 2012), (Sayin, 2010, 2011b, 2012a-bch, 2014, 2015). 
7) Las mujeres con RSE describieron zonas erógenas sensibles en sus genitales distintos del clítoris (Morris, 2004), (Armagan, 2012), (Sayin, 2012a-bch, 2014, 2015).

8) Las mujeres con RSE se masturbaban con mayor frecuencia en comparación con las mujeres con NRSE (Armagan, 2012), (Sayin, 2012a-eh, 2014).

9) Las mujeres con RSE tenían fantasías eróticas con más frecuencia que las mujeres NRSE (Armagan, 2012), (Sayin, 2012a-eh, 2015).

10) Las mujeres con RSE admitieron tener una forma de estados alterados de conciencia durante algunos de sus orgasmos prolongados y / o status orgasmus (Taylor, 2000, 2002), (Mah, 2001, 2002, 2005), (King, 2010), (Sayin, 2011c; Sayin, 2012a-dh, 2015).

\section{Zonas erógenas vaginales profundas -DVZ-}

Después de investigar los orgasmos de las mujeres durante 25 años, debemos admitir que "el orgasmo de cada mujer es único y pertenece a ella"; en otras palabras, hay tantos patrones de orgasmos como el número de mujeres existentes en el mundo.

Las mujeres difieren de muchas maneras en términos de sus respuestas a los estimulos; sus cuerpos sexuales, cerebros sexuales y sus respuestas genitales son muy diferentes entre sí y no se puede definir un estereotipo estándar de orgasmos que sea válido para cada mujer.

Hay muchos parámetros que influyen en los patrones de orgasmo de las mujeres, como la psicología, el aporte subconsciente, el inconsciente colectivo, la libido, el deseo sexual, los niveles de testosterona libre en la sangre y de la estructura hormonal general, antecedentes psicológicos, imaginación, experiencias anteriores, coeficiente intelectual, cociente 
emocional, cociente sexual, conjunto y entorno, las capacidades de la pareja, traumas e incidencias buenas o malas.

Debemos admitir otro hecho de que si la mujer experimenta orgasmos vaginales, cuyo mecanismo es muy diferente de los orgasmos del clitoris, ella es más probable que mejore sus patrones orgásmicos y desarrolle RSE. Los orgasmos vaginales se correlacionan con otras zonas erógenas, que no sean el glande del clitoris, que se han definido recientemente y acuñadas como Zonas erógenas vaginales profundas -DVZ- (Morris, 2004), (Levin, 2014), (Chua, 1997), (Sayin, 2012-h, 2014, 2015), (Zdrok, 2004).

Hemos investigado la posible existencia de tales áreas en nuestras encuestas y otros proyectos de investigación y encontramos la descripción de áreas tan sensibles que pueden contribuir al desarrollo del orgasmo femenino en una minoría de mujeres (Sayin, 2011 abcd, 2012a-bch, 2014-a, 2015).

Las descripciones de las DVZ y la frecuencia de aparición de la conciencia de las DVZ en algunas mujeres ${ }^{2}$, particularmente con RSE, fueron las siguientes (ver figura 2):

G-SPOT: (Mancha de Grafenberg): la localización del punto G se encuentra en la pared vaginal anterior, $2.5-4 \mathrm{~cm}$ en el interior, debajo de la longitud uretral media. En nuestra serie, el $31.8 \%$ de las mujeres admitieron estar al tanto de sus G-Spots. E1 27.8\% de ellas estaban seguras de haber experimentado orgasmos en el punto G. El $45,4 \%$ de estas mujeres eran mujeres con RSE.

${ }^{2}$ Grupo total $n=198:$ Mujeres con RSE $n=35$ y mujeres sin control de RSE $n=163$ 
A-SPOT: Está en la pared anterior de la vagina, $2-3.5 \mathrm{~cm}$ debajo del fórnix anterior, debajo de la vejiga. El 10,6\% de las mujeres admitió estar al tanto de dicha zona erógena. El 61,9\% de ellos eran mujeres con RSE.

O-SPOT: Se encuentra entre la pared vaginal posterior y el recto, 2-4 cm por debajo del fórnix posterior. El 8\% de las mujeres respondieron que tienen un área sensible en esta parte de sus genitales. E1 75\% de ellos eran mujeres con RSE.

Cervix: Es el collum -cuello- del útero. El 7,5\% de las mujeres respondió que su cuello uterino era sensible y podría haber desencadenado un orgasmo. El 60\% de ellos eran mujeres con RSE.

Músculos del piso pélvico: (PFM-PC-Muscles): los PFM son la red muscular entre el pubis y el cóccix. $12.1 \%$ de las mujeres dijeron que la activación de PFM era efectiva para el desarrollo de un orgasmo. E1 50\% de ellos eran mujeres con RSE.

La mayoría de las mujeres con RSE admitieron que pueden tener zonas erógenas como DVZ, que pueden participar en el desarrollo de un orgasmo, que no sea el glande del clítoris. En nuestro estudio preliminar en 198 mujeres, algunos de los puntos DVZ fueron identificados por 35 mujeres con RSE y 163 mujeres NRSE (Sayin, 2012a-bcdeh, 2014-b, 2015). 
A

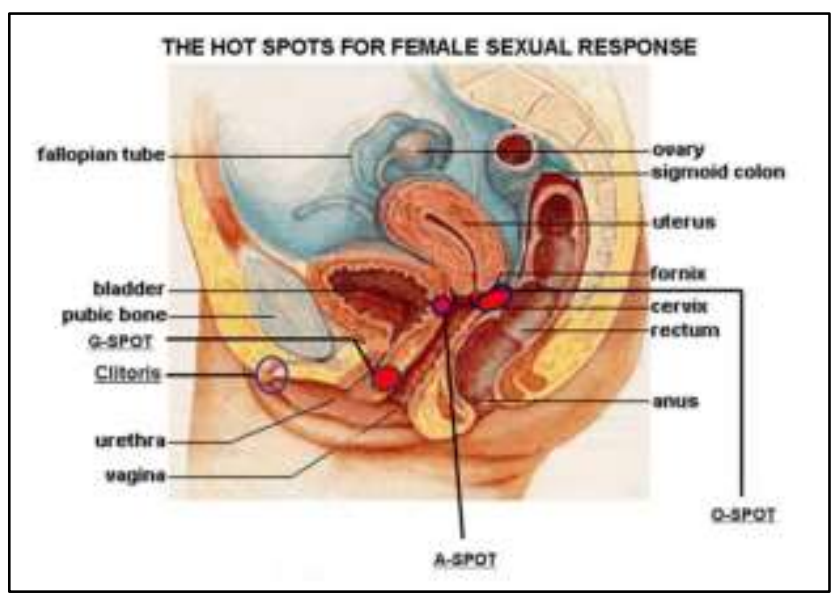

$\mathrm{B}$

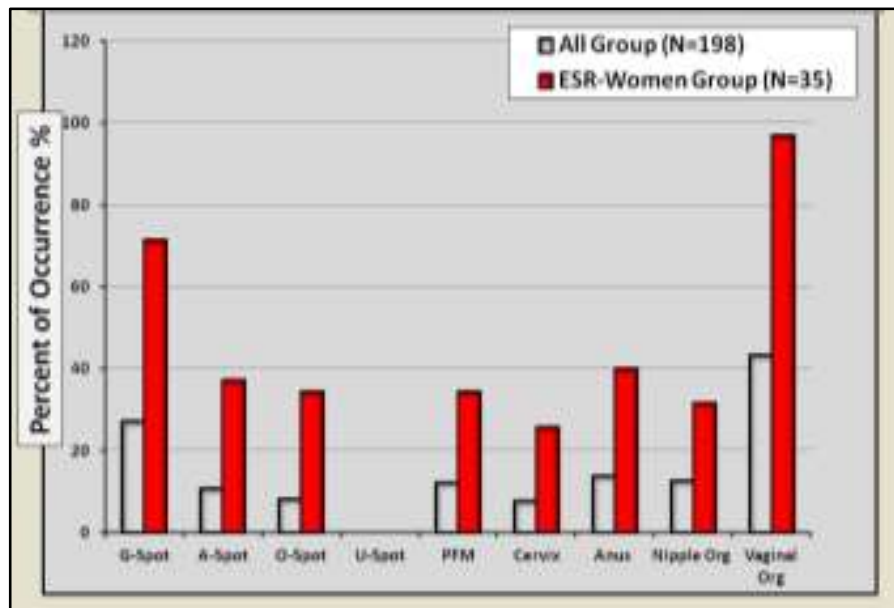

Figura 2

A) Anatomía de las zonas erógenas vaginales profundas (DVZ).

B) La distribución de los porcentajes de las áreas DVZ: La investigación de DVZ entre 198 mujeres junto con la investigación de la encuesta sobre RSE. El cuestionario de la encuesta tenía descripciones detalladas y figuras esquemáticas de DVZ. El número total de 198 también incluye a 35 mujeres con RSE. Como se ve en la gráfica, la frecuencia de la presencia de DVZ fue mucho mayor en mujeres con RSE, que alcanzaron orgasmos vaginales en casi el 99\%. Algunas mujeres admitieron que experimentaron orgasmos a través de la estimulación de G-Spot, A-Spot, O-Spot, Anus, PFM, Cervix, Nipples, sin embargo, nadie tenía un recuerdo de placer u orgasmo por la estimulación de las coordenadas de un área eso coincidió con "el hipotético U-Spot", como lo describió Morris en 2004. 


\section{Dos muestras de orgasmos tántricos con RSE}

Seguir dos muestras de la descripción de un orgasmo de RSE que hemos registrado en nuestras encuestas puede aclarar y visualizar la "conciencia orgásmica" de una mujer con RSE (Sayin, 2010, 2012-h, 2015); estos dos casos no tenían ningún trastorno psiquiátrico o no fueron tratadas por ningún trastorno psiquiátrico; ni sufrian de PSAS o PGAS -síndrome de excitación genital persistente-:

\section{Caso A:}

JK era médica. Durante la entrevista tenía 33 años, hoy tiene 38 años. Tuvo su primera relación sexual a los 16 años, tuvo su primer orgasmo vaginal a los 24 años, cuando también descubrió su Punto G. Comenzó a tener orgasmos expandidos y status orgasmus a la edad de 28 años. Experimentó varias formas de ASC durante orgasmos prolongados.

Tuvo casi 40-50 parejas desde que tenía 16 años. Su puntaje de la escala RSE fue 132/150 en 2011. Ella dice que ha sido hipersexual desde que tenía 26 años. Agrega que ha estado practicando ejercicios de Kegel desde que tenía 26 años. Experimentó sensaciones sensuales y estimulación en el punto $\mathrm{G}$, punto $\mathrm{A}$, punto $\mathrm{O}$ y tenía orgasmos del punto G y eyaculación femenina.

Se definió a sí misma como "hiperactiva en sexualidad y respuesta orgásmica", pero agregó que no era "una adicta al sexo"; ella estaba contenta con una breve actividad sexual y estaba muy contenta con su vida sexual. La mayoría de sus relaciones eran monógamas.

Sus impulsos sexuales no eran incontrolables; ella habia tenido algunos períodos de celibato en su vida. El mayor número de orgasmos, como ella definió, fue de 25 a 30 orgasmos múltiples por hora; ella 
experimentó orgasmos que duraron por un par de horas continuamente. No ha sido diagnosticada y / o tratada por ningún trastorno psiquiátrico:

JK definió sus orgasmos del siguiente modo:

"Durante mis orgasmos me alejo de todo, es una despersonalización total. Solo me siento, incluso me olvido de mi misma. Solo se quedan mi voz y mis gritos. En el status orgasmus, que es lo mejor que puedo disfrutar, el placer aumenta gradualmente, estoy totalmente aislada de mi entorno.

Solo escucho la voz animal que sale de mi garganta, mis gemidos cortos resultan ser gritos increíbles. Lo siento en el estómago, primero como un hormigueo, luego las contracciones se suceden. Es un estado de conciencia totalmente alterado.

Mi visión se oscurece. Veo destellos de colores o luces. Durante orgasmos menores siento contracciones divertidas, como un juego. Los orgasmos o contracciones menores que comienzan en mi vagina $y$ pubis circulan a través de mi estómago, donde puedo sentir el centro real del volcán orgásmico.

Una vez que comienza el status orgasmus, es como un huracán que me aleja de mi cuerpo y vuelo, estos orgasmos menores, cada uno con una duración de casi 10 a 20 segundos, se acumulan en un ataque continuo, mientras que a veces no puedo escuchar mis gritos. Estoy perdida en los primeros minutos.

Estas contracciones continúan durante 10-20 minutos, especialmente cuando mi pareja está haciendo sexo oral conmigo. Si continuamente estimula mi clitoris con su lengua un orgasmo termina y otro comienza. 
Durante el status orgasmus siento que estoy viajando por el mundo, como si tuviera un cuerpo astral, voy a jardines desconocidos, cascadas, prados. Durante los orgasmos en las relaciones sexuales siento una increíble unificación y fusión, y se disuelven el uno en el otro. También tuve status orgasmus durante las relaciones sexuales.

Si mi pareja continúa teniendo relaciones sexuales durante 1-3 horas, es fácil alcanzar orgasmos prolongados. Mi cerebro se derrite, me doy cuenta de que soy un animal. Oigo las voces de mis animales saliendo de mi garganta que me excitan más. Los orgasmos vaginales a veces son mejores que el del clitoris.

Cuando utilizamos un vibrador durante la relación sexual, entro fácilmente en un status orgasmus que dura de diez minutos a media hora, mientras que experimento casi 50-60 orgasmos menores.

Durante los orgasmos, me rio, lloro, gimo, hago ruidos muy fuertes isiempre tengo miedo de que los vecinos puedan oírme! Mi cuerpo se arquea, estoy en plena extensión, a veces no estoy segura si tengo dolor o placer. Las fantasias susurradas en mi oído me vuelven loca durante estos orgasmos.

Incluso una palabra puede iniciar otro orgasmo menor. Podria ser cualquier cosa, salvaje o suave. Quiero convertirme en un animal, volver a mi cuerpo arquetípico, sea lo que sea. Cuando me contraigo me siento como un animal, como si viniera de mi subconsciencia colectiva. Siento el pene como una espada ardiente que me marca.

Hace que mi pelvis esté cada vez más caliente, se evapora y luego se produce una gran contracción que sigue a otra. El status orgasmus es muy satisfactorio para mí, en comparación con otros orgasmos, como los debidos solo a la estimulación del clitoris o la vagina. 
Me siento tan mareada y mi cerebro está tan alto y girando que creo que seria muy dificil para mí caminar en linea recta, me caería. Me encanta perderme en mi propia química cerebral. No estaría satisfecha si no experimentara el status orgasmus, porque los orgasmos menores del clitoris o la vagina son muy bajos para mí.

No me hacen tan feliz como el status orgasmus. No tengo ningún periodo refractario para alcanzar orgasmos vaginales o del clitoris. Uno puede venir después de otro sin convertirse en un status orgasmus. Pero para el status orgasmus, siempre hay un periodo refractario de 15-20 minutos..."

\section{Caso B:}

IAY es ingeniera. Tenía 34 años, hoy 35. Tuvo su primera relación sexual a los 20 años. Tuvo sus primeros orgasmos en el clitoris a edades muy tempranas, desde los 8 hasta los 10 años, por medio de la auto masturbación. Tuvo sus primeros orgasmos vaginales a los 24 años y su primer orgasmo combinado con status orgasmus a los 32 años. Su puntaje de la escala RSE fue 142/150 en 2015.

Sus músculos PC eran muy fuertes, de modo que sus músculos PC podian tener una presión de 25-30 mmHg, usando una sonda Kegel o más. Sus orgasmos fueron, la mayoria de las veces, múltiples y experimentó un status orgasmus que duró un par de horas, siempre y cuando fuera estimulada.

La mayor duración de sus orgasmos múltiples, según recordaba y expresó, fue de 8 a 10 horas, de forma continua con una pareja en particular; con coito, estimulación del clítoris, sexo oral, vibraciones y juguetes, y otras técnicas tántricas. Su patrón de orgasmo más alto 
reportado fue de 35 a 45 orgasmos continuos en una hora, que también duró continuamente durante un par de horas.

Experimentó una sensación sensual y estimulación en G-Spot, A-Spot, O-spot; y experimentó orgasmos del punto G y eyaculación femenina. Se definió a sí misma como "hipersexual", pero no como "adicta al sexo o insaciable".

Sus impulsos sexuales no eran incontrolables; ella tuvo algunos períodos de celibato muy largos en su vida, sin ninguna interacción sexual.

La mayoria de las veces era monógama y estaba muy contenta con su vida sexual y sus orgasmos. No ha sido diagnosticada y / o tratada por ningún trastorno psiquiátrico.

IAY definió sus orgasmos del siguiente modo:

«Me gustaria explicar mi orgasmo, explicar mis experiencias orgásmicas en diferentes formas de orgasmos:

Mis orgasmos en el clitoris: cuando la sensación de hormigueo comienza en mi clitoris después de la estimulación manual o por vibración, siempre tengo una sensación de cosquilleo y esta sensación se extiende a mi pelvis, mis piernas y mi torso gradualmente con la creciente cantidad de placer, que se acumula arriba.

Me siento inmersa y fluida, elevada, cuando comienza el orgasmo. En general, vengo con mucha facilidad y la estimulación continua de mi clitoris con dedos lubricados, lengua o vibraciones me hace comenzar a venir continuamente mientras mi cuerpo y genitales se contraen $y$ siento la explosión en mi útero. 
Mis piernas $y$ mis genitales $y$ pelvis siempre tiemblan, y no puedo evitarlo. Este temblor se convierte en una especie de terremoto $y$ finalmente se convierte en un ataque epiléptico. Los músculos de mis piernas se contraen y se relajan con cada orgasmo. Las plantas de mis pies se calientan mucho, mientras que este calor enreda mi torso y mi cuerpo gradualmente. Mis genitales y el clitoris palpitan y siempre esta sensación palpitante con el ritmo del corazón son las únicas cosas que recuerdo, cuando llego a 20-30 orgasmos si continúa la estimulación oral, manual o vibratoria.

Siento descargas eléctricas y explosiones en mi clitoris y otras partes de los genitales. Los orgasmos del clitoris pueden volver sucesivamente, pero son explosivos, locales y no son tan satisfactorios como los orgasmos vaginales; Los orgasmos vaginales se extienden por todo el cuerpo y el cerebro, a diferencia de los orgasmos del clitoris.

Después de diez orgasmos, mi mente se va, si los orgasmos continúan no puedo hablar, solo escucho mis gemidos y gritos o risas, lo que también aumenta mi placer y mejora los próximos orgasmos. En resumen, el orgasmo del clitoris es como un orgasmo agudo, corto, explosivo con descargas eléctricas repentinas. Puedo continuar con los orgasmos del clitoris durante un par de horas con una pareja sin descanso y sin cesar.

El mayor número de orgasmos en el clitoris que tuve en una sesión por masturbación o por la estimulación de una pareja fue probablemente más de cien en un par de horas.

Mis orgasmos vaginales: son mucho más satisfactorios $y$ se extienden a todo mi cuerpo. Después de tener 15-20 orgasmos del clitoris, es mejor pasar al coito y a los orgasmos vaginales. Porque hay más excitación entonces. Siento mi punto G desde los primeros tiempos 
de las sesiones de coito desde que tenía 21-22 años. También tuve una experiencia de orgasmos del punto $G$ y orgasmos vaginales por separado, mientras que también eyaculaba ocasionalmente.

Durante el coito siento que mi punto $G$ se agranda $y$ da placer en niveles muy altos. Mi placer A-Spot y Cervical es tan poderoso como la estimulación del G-Spot. Entonces, cuando mi pareja puede continuar con el coito durante 60 a 90 minutos, puedo sentir fácilmente la estimulación de G-Spot, Cervix y A-Spot por separado; después de un tiempo se combinan en orgasmos enormes muy intensos $y$ hermosos.

En cada variedad del pene, en cada longitud hay un tipo diferente $y$ sabor de placer que se acumula gradualmente. Después de un tiempo no puedo diferenciar un orgasmo de otro; uno comienza y continúa con las contracciones de mis genitales, útero y cuerpo, cada vez más intenso. Cuando termina, puede comenzar otro orgasmo dependiendo de los juegos previos, las conversaciones y las fantasias contadas por la pareja.

Cuando aprieto los músculos PC, la intensidad de los orgasmos siempre mejora. Por ejemplo, apretar los músculos del PC tiene más efectos sobre el placer aumentado de G-Spot. Puedo llegar al orgasmo con la estimulación única de G-Spot, o A-Spot y / o Cervix. Siento el O-Spot con menos frecuencia, pero soy consciente de que también hay un lugar de placer en la parte posterior de mi vagina.

Durante el orgasmo vaginal, si continúan durante 30-40 minutos con diferentes intensidades de explosiones, me unifico con mi pareja y mi mente y estado de ánimo se elevan; es como morir y experimentar fuera del cuerpo, como observar el cuerpo desde afuera. 
Me convierto en personas diferentes y como si hubiera otras personas dentro de mí, como una criatura de la libido, que sale y desea solo ser completada y experimentar coito prolongado por más y más... Durante los orgasmos vaginales experimento estados alterados de conciencia. Los orgasmos vaginales, cuando la pareja es lo suficientemente buena como para estimular, se acumulan en formas increibles de contracciones que son más intensas y profundas y más largas que los orgasmos del clitoris.

Los orgasmos vaginales son mucho más satisfactorios en comparación con los orgasmos del clitoris. El orgasmo vaginal es como subir a una montaña, cuando llegas cerca de la cima de la montaña, me siento como trenes continuos de orgasmos que se mejoran mutuamente. Los vibradores rotativos en el clitoris también inducen orgasmos muy poderosos en mis encuentros sexuales.

Una vez continué teniendo orgasmos vaginales + clitoris hasta que se apagaron las baterias del vibrador rotativo, no recuerdo cuántos minutos $u$ horas fueron.

Orgasmos múltiples: nunca tengo orgasmos individuales. Siempre son múltiples y antes de sentir alivio, necesito tener de 15 a 25 orgasmos múltiples en el clitoris o la vagina. Entonces, una vez que pase 25-30, el patrón del orgasmo puede cambiar y mi respiración se detiene, mi mente se detiene, siento que me estoy muriendo y los trenes de orgasmos se convierten en un status orgasmus.

Orgasmo mixto y status orgasmus: a pesar de que experimenté orgasmos múltiples en el clitoris y la vagina muchas veces, aprendi orgasmos mixtos $y$ status orgasmus hace un año y medio con una nueva pareja que podia continuar el coito y estimularme de muchas maneras durante un par de horas 
Era un amante tántrico y antes que él, todos mis compañeros en mi vida podian continuar el coito o la estimulación durante 20 a 30 minutos a lo sumo. La pareja es muy importante para experimentar orgasmos vaginales, orgasmos mezclados y status orgasmus.

La explosión en los orgasmos y el estado combinados es inmensa y es como subir una escalera de infinitos escalones, donde en cada escalón el orgasmo es más intenso que el anterior. En status orgasmus, siento que cada célula mía tiene un orgasmo. En mis experiencias, la combinación de la estimulación del clitoris y el A-Spot crea orgasmos magnificos, que son indefinibles.

Mi cuerpo se arquea, grito como si algo me estuviera torturando o matando. Las contracciones son continuas y la mejora gradual del orgasmo nunca termina, mientras eyaculo demasiado, y las sábanas se empapan tanto que es como un río que fluye desde mi útero hasta la vagina y luego afuera.

Status orgasmus: es el nirvana sexual para mi. Es una gran iluminación y satisfacción que no puedo explicar y dilucidar el placer y la inmovilidad que obtengo después de un orgasmo de estado. Si la pareja es buena, puedo alcanzar el status orgasmus a través del orgasmo vaginal, que se convierte en un status orgasmus de un tiempo.

Pero, la mayoria de las veces, la estimulación combinada de G-Spot, A-Spot, coito $y$ clitoris me pone en status orgasmus en 10 minutos. Luego estoy en otro mundo, el paraiso del placer y las contracciones, perdiéndome y solo me concentro en mis gritos, contrayendo genitales y calentando y contrayendo las piernas.

En cada orgasmo combinado o status orgasmus, experimento varias formas inesperadas e inusuales de ASC escritas en su Escala de 
RSE. El mundo exterior se cierra y me convierto en una criatura orgásmica diferente. Solo me siento a mí misma, mi cuerpo continuamente contraido y mi pareja; nos unificamos. Solo pienso en venir más y más y más. Después de 15-20 minutos de status orgasmus continuo, una criatura o un animal sale de mi mente $y$ cuerpo.

Ese animal vive solo por placer y pide más climax, solo está en mi cuerpo por coito $y$ dice $f . . k$.; $f \ldots k ; f \ldots k$. Aunque mi compañero salga de mi vagina, todavía lo siento adentro y los orgasmos continúan.

Este es un verdadero pico y una experiencia extraordinaria. Luego me convierto en una máquina de orgasmo y, como una ametralladora de orgasmo, vengo y vengo con mayores intensidades en cada momento... iSiento solo mi cuerpo contraído, incluso en cada órgano, cada tejido y cada célula! Mi pareja a veces tiene miedo de mi estado de ánimo y expresiones faciales, porque no puedo hablar, no puedo emitir un sonido y no puedo respirar.

Cuando se detiene el status orgasmus, me siento como un pedazo de cuerpo en mal estado, no puedo hablar durante casi 15-20 minutos, me acuesto y no puedo moverme mientras todavia muchas partes de los músculos y genitales de mi cuerpo continúan contrayéndose. El placer es tan inmenso e inexplicable que nunca experimenté tal alteración de mi estado de ánimo y mi cuerpo orgásmico. Es una especie de conciencia diferente del orgasmo.

\section{Estados alterados de conciencia durante orgasmos combinados}

o status orgasmus: Siento más ASC que lo que está escrito en su Escala de RSE. Todo tipo de alucinaciones cortas en la cima del estado. Tranquilidad, flotar, volar, experiencia fuera del cuerpo, morir, explotar, viajar en el tiempo. Viajar y volar sobre bosques y ciudades 
desconocidas, viajes astrales como experiencia, elevarse, llorar o reír, risas espontáneas, volar como un caballo alado, todo tipo de sentimientos de libertad, sentimientos oceánicos y de felicidad, sentirse como una mariposa y un pájaro sagrado y mitológico que vuela sobre los cielos azules y las tierras. Mi amor a mi pareja aumenta. Siento tantas cosas diferentes que siento todos los ASC en la escala RSE más muchos otros..."

\section{Algunas caracteristicas de las mujeres con RSE:}

Algunas de las características promedio de las mujeres con RSE fueron las siguientes (Sayin y Kocatürk, 2012-j):

1) Tenían la capacidad de excitarse más fácilmente.

2) Tenían una libido muy elevada.

3) Eran muy conscientes, conscientes y sensibles al Punto G, DVZ y otras zonas erógenas distintas del glande del clitoris. Sus umbrales de excitación a estímulos sexuales disminuyeron en respuesta a vibradores (50-200 Hz), coito, sexo oral, estimulación manual y / u otros métodos como caricias, toques, estímulos labiales.

4) Pasaron más tiempo en cuestiones sexuales.

5) Tenían más fantasias y más tendencia a tener variaciones sexuales, desde variaciones suaves hasta extremas. Muchas mujeres hiperactivas que entrevistamos tenian muchos patrones de fantasía diferentes, desde suaves hasta salvajes y extremos, aunque nunca experimentaron ninguno de ellos (Sayin, 2014, 2016).

6) Generalmente usaban juguetes sexuales y vibradores también. 
7) Se masturbaban con mayor frecuencia incluso en presencia de una pareja. Su frecuencia de masturbación aumentó cuando no tenían pareja.

8) Su cerebro sexual y psicología sexual estaban más desarrollados y receptivos. Algunas pueden haber experimentado "orgasmos cerebrales" simplemente fantaseando y usando PFM, PC-Muscles.

9) Eran menos inhibidas, más provocativas y promiscuas. Eran muy permisivas y liberadas. Su mente estaba más abierta a los asuntos y novedades sexuales.

10) Tenian más experiencia en el sexo, tenían más parejas y relaciones sexuales más largas. Sin embargo, el número de parejas no fue un problema, como señalaron muchas de ellas. La calidad de la relación y del sexo era más importante que la cantidad. No eran mujeres en busca de nuevas parejas todas las noches, para una noche; sin embargo, prefirieron relaciones monógamas satisfactorias y de largo plazo.

11) Su imaginación, IQ e inteligencia emocional parecian ser más altos y más desarrollados. Prefirieron estar en relaciones más profundas y de tipo alma gemela con los hombres que en las superficiales.

12) Las mujeres con RSE parecian creer menos en términos de prácticas religiosas tradicionales en comparación con las mujeres con NRSE, mientras que tenían poco o nada de dogmas culturales y religiosos.

13) Algunas mujeres con RSE informaron tener eyaculación durante el orgasmo como el hombre, un fenómeno que había sido reportado por Ladas, Perry y Whipple (Ladas, 1982). 
14) Las mujeres con RSE experimentaron estados alterados de conciencia durante el orgasmo prolongado con mayor frecuencia que las mujeres con NRSE. El número de variación de diferentes sentimientos subjetivos y ASC de las mujeres con RSE, durante las diferentes formas de orgasmos y orgasmos prolongados de RSE, fue mucho mayor en comparación con las mujeres con NRSE (Taylor, 2000), (Sayin, 2011), (King, 2010).

15) Las mujeres con RSE tenían estilos de vida sexual más felices, contentos y satisfactorios en comparación con las mujeres con NRSE. Ninguna mujer con RSE acudió a un psicólogo clínico o psiquiatra por cualquier queja de disfunción sexual, por lo que la existencia del fenómeno de RSE y las mujeres con RSE no se han identificado y discutido en la literatura médica y la literatura de psiquiatría.

16) Las mujeres con RSE pueden haber experimentado patrones de orgasmo muy largos, múltiples, prolongados y sostenidos y también el fenómeno llamado status orgasmus, que duró de 1-2 minutos a 10-15 minutos o más (Sayin, 2010, 2011, 2012, 2013, 2014, 2015), (Taylor, 2002), (Schwartz, 1999).

\section{Limites conocidos del orgasmo femenino}

Los limites conocidos del orgasmo femenino pasados a los libros de registro fueron 134 orgasmos por hora; Este registro pertenecía a una mujer ciclista. Después de ella, este récord de orgasmo femenino se elevó a casi 200 orgasmos por hora reportados por una fuente india (Sayin, 2014).

Estas mujeres no sufrían de PSAS o PGAS -síndrome de excitación genital persistente -. Durante el PGAS, se informó que algunas mujeres 
con PGAS o PSAS experimentaron orgasmos espontáneos de 50 o 100 o más orgasmos durante un par de horas, que fueron espontáneos, incontrolables y patológicos.

Estas cifras demuestran que algunas mujeres tienen el potencial de experimentar un par de orgasmos por minuto, que pueden continuar durante decenas de minutos $u$ horas, sin una fase de resolución o un período refractario, como se ha informado en la literatura antigua del Lejano Oriente.

La Dra. Patricia Taylor también ha demostrado que la intensidad, el número y la duración de los orgasmos pueden extenderse y expandirse más allá de los límites conocidos (Taylor, 2000, 2002, 2004). Acerca de esto, tiene un video de sus experiencias que también demostró que esto era posible (Taylor, 2004).

\section{Discusión}

Aunque los orgasmos prolongados se habian definido en las antiguas culturas y literatura pagana, chamán, tántrica y taoísta, tal enfoque en Occidente ha sido aceptado como inmanejable e inimaginable durante siglos.

Esta comprensión fue parcialmente el resultado de los prejuicios de algunos de los sistemas de creencias monoteístas, que eran altamente antropomórficos, autocráticos y dominados por los hombres.

En las culturas orientales, las mujeres eran consideradas como parte de las Diosas y lo divino, mientras que en occidente, durante siglos, las mujeres eran consideradas como un medio del pecado universal de la humanidad, mientras que cualquier cosa relacionada con la sexualidad era mala, diabólica y de temer. 
Por lo tanto, los occidentales ni siquiera se molestaron en investigar los posibles limites del potencial de las mujeres. Incluso, hasta la traducción del Kama Sutra al inglés, nadie cuestionó la importancia de las posiciones sexuales.

Mientras que en el catolicismo la única posición sugerida fue la posición misionera durante cientos de años, porque otro tipo de posiciones podría inducir la estimulación del glande del clítoris, el punto G y DVZ y podrian dar placer a las mujeres, los templos de la cultura tántrica son estatuas y relieves completos de dioses y diosas, con coitos de muchos estilos diferentes, a pesar de que las principales religiones del hinduismo y el budismo también consideraban que la sexualidad y el placer eran algo de lo que debía ser cauteloso y mantenerse alejado.

Por lo tanto, en Europa, todo lo relacionado con la sexualidad estaba prohibido y a las mujeres no se les permitía disfrutar del sexo y alcanzar el clímax. En las culturas tántricas, por el contrario, las mujeres fueron entrenadas para dar placer y disfrutar de las actividades sexuales.

Por ejemplo, el entrenamiento tántrico de los músculos PC -PFM, músculos del piso pélvico- y el entrenamiento chino de estos músculos mediante el uso de bolas de Ben-Waa desde la pubertad se consideraron esenciales para una buena salud sexual, así como posiciones sexuales, meditación y concentración, ejercicios de respiración, ejercicios de enfoque sensorial, masaje sensual, rituales prolongados, coito prolongado (Vatsyayana, 1883), (Chang 1977, 1983), (Wu, 1996), (Schwartz, 1999), (Chia, 2002, 2005), (Tannahill, 1992), (Muchembled, 2008).

Los hombres y mujeres en Occidente comenzaron a redescubrir la sexualidad durante los días de la Revolución Sexual Occidental y el Movimiento de la Nueva Era, después de la Segunda Guerra Mundial, 
cuando se comenzó a investigar la sexualidad humana sin prejuicios y con métodos científicos objetivos y racionales.

Fue entonces, cuando la mente occidental se dio cuenta de que los orgasmos femeninos prolongados y la actividad sexual prolongada podrian ser posibles, después de aprenderlo de las culturas orientales.

La literatura china antigua que proviene del siglo I a. C. describe el orgasmo femenino, así como los escritos hindúes en los siglos II y III, como el Kama Sutra, de los siglos VI-VII (Vatsyayana, 1883), (Chang 1977, 1983), (Wu, 1996), (Schwartz, 1999), (Chia, 2002, 2005), (Mumford, 2005), (Michaels, 2008), (Muchembled, 2008), (Morris, 2004).

El orgasmo femenino fue definido por primera vez en los siglos XIX y $\mathrm{XX}$ por algunos pioneros de la investigación sexual en Occidente (Ellis, 1897), (Freud, 1905), (Reich, 1973), (Masters \& Johnson, 1966), (Fisher, 1972-1977), (Escapa, 1989), (Muchembled, 2008), (Sayin, 2014).

En realidad, el término "orgasmo" proviene de los siglos V al IV antes de Cristo, viniendo del término "orgia», durante los tiempos paganos. Orgia fue un festival del Culto Dionisiaco, una encarnación de los mitos griego-helénicos en la forma de festivales Orgia -primavera $u$ otoño - en la secreta y selecta ciudad de Eleusis (Ruck, 2006). Orgia, con el tiempo tomó la forma de Orgasmo.

\section{Posibilidad de alcanzar otros limites}

El placer y alcanzar el clímax sexual, es un fenómeno aprendido. La sensualidad, el cerebro sexual, el cuerpo sexual, la sensibilidad en los genitales y los reflejos del orgasmo también se pueden desarrollar y mejorar a través de ejercicios y entrenamiento. 
Durante nuestras encuestas de 25 años entre mujeres y durante los talleres de RSE y Neo-Tantra, hemos observado que la expansión del conocimiento sobre el cuerpo humano, la fisiología sexual, los ejercicios tántricos y otras experiencias también, con el tiempo, aumentan la sensualidad, sensibilidad y capacidad para alcanzar mejores orgasmos en muchas mujeres.

Alcanzar orgasmos mejorados e incluso RSE se puede aprender y entrenar. Para dar un ejemplo, una mujer que es sexualmente receptiva -con reflejos de orgasmo vaginal y del clitoris- y que alcanza 5 orgasmos múltiples en 30 minutos, puede recibir capacitación de tal manera que, después de un tiempo, pueda comenzar a tener 15 orgasmos múltiples en 20 minutos; o 40 orgasmos en una hora.

Aquí, lo más crucial es la capacidad y el enfoque de su pareja; su pareja también debe ser entrenada con ella. Hoy, la RSE y el status orgasmus se pueden verificar en menos del 10\% y menos del 1\% de la población femenina, respectivamente.

En otras palabras, las mujeres que están entrenadas en RSE y NeoTantra no deben esperar alcanzar un nivel de RSE, que es exclusivo de alguna minoría de mujeres.

\section{Conciencia Orgásmica y Orgasmos Tántricos}

Algunos de los estados alterados de conciencia durante la respuesta orgásmica femenina prolongada, de la más leve a la más severa, fueron (King, 2010), (Mah, 2001), (Sayin, 2011-c, 2012-ah): 
- Sensación de pulsación.

- Sensación palpitante.

-Euforia.

- Estremecimiento.

- Sensación de absorción.

- Sensación de disolverse.

- Sensación de fluidez.

- Sensación de chorrear.

- Sentimiento irreal.

- Sentimiento surrealista.

- Elevación del estado de ánimo.

- Aumento del entusiasmo y la creatividad.

-Explosión.

- Ver luces encendidas

-Ver diferentes objetos geométricos.

- De ja vu

- Sensación de terremoto.
•Volcán.

-Éxtasis

- Vuelo, volar.

-Despersonalización.

- Perderse.

- Experiencia fuera del cuerpo.

- Perder el sentimiento del alma.

- Experiencia cercana a la muerte.

- Sentimiento de muerte.

- Unificarse, sentimiento unificado.

- Unificarse con el entorno y el universo.

- Cese del tiempo, el tiempo se detiene.

- Distorsión en el continuo espaciotiempo.

- Experiencia mística.

Figura 3:

Algunos patrones llamativos de estados alterados de conciencia (ASC) durante orgasmos normales y orgasmos prolongados.

El orgasmo femenino se ha descrito como un estado de ASC en la literatura (Davidson, 1980), (Komisaruk, 2006), (Sukel, 2011), (Sayin, 2012a-d, 2014-a, 2015).

Durante los orgasmos prolongados, que pueden durar decenas de minutos, algunas minorias de mujeres pueden alcanzar un estado 
diferente de conciencia, éxtasis y sentimientos o estados de ánimo divino, oceánico y feliz.

La psico-neurofarmacología del orgasmo femenino tiene una especie de efecto "corto alucinógeno" en las mentes de las mujeres. Algunas mujeres lo llaman "Nirvana sexual" o "Satori 3 , con sentimientos de éxtasis y unificación. En la literatura tántrica, también se describe que se puede lograr un tipo de estado de nirvana a través de la sexualidad prolongada y los orgasmos (Carellas, 2007), (Wu, 1996), (Chia 2002, 2005), (Mumford, 2005), (Michaels 2008).

Los términos "orgasmo tántrico" u "orgasmo cósmico" se mencionan con frecuencia en libros de Tantra. Por lo tanto, la sensualidad y el climax mejorado y prolongado, pueden considerarse un medio para alcanzar algún tipo de experiencias psi.

\section{Conclusión}

Según el conocimiento antiguo y arcaico, las mujeres pueden experimentar estados y conciencia orgásmicos inusuales y extremos, a través de la estimulación sexual continua, que dura decenas de minutos u horas.

Para el mundo occidental, este fenómeno es muy extraño y nuevo. La ciencia puede desentrañar muchos otros hechos nuevos sobre la sexualidad masculina y femenina. La combinación de antiguas técnicas tántricas y taoístas, con el poder de la ciencia moderna puede conducir a descubrir nuevos horizontes en la sexualidad tanto para hombres como para mujeres.

\footnotetext{
${ }^{3}$ Satori es un momento de no-mente y de presencia total, término japonés que designa la iluminación en el budismo zen. La palabra significa literalmente comprensión
} 
Como occidentales, estamos en la etapa de unificar el viejo conocimiento de la sabiduría con el conocimiento científico objetivo y racional. Por lo tanto, los aspectos tántricos y taoístas y las técnicas de sexualidad mejorada también deberian ser un objetivo para que la ciencia moderna investigue a fondo. En realidad, la terapia sexual y la sexología, hoy en día, solo investigan los trastornos de la función sexual y la sexualidad patológica. El objetivo principal de la terapia sexual no debe ser solo investigar sobre los trastornos y enfermedades de la sexualidad, sino también realizar una investigación novedosa para desentrañar los secretos de la sexualidad humana y descubrir cómo mejorar el placer sexual y los orgasmos -en particular de las mujeres- para un mejor desempeño de parejas.

Como académicos y científicos, no debemos dejar las técnicas tántricas en manos de charlatanes y traficantes de sexo, que utilizan los términos "Tantra" y "Neo-tantra" para abusar sexual y emocionalmente de sus incautos "discipulos", como ocurre en la India y otros lugares, de modo que a este artículo le deberán seguir nuevas investigaciones multidisciplinarias.

Créditos: Traducción del original en inglés publicado en la Revista Científica Arbitrada de la Fundación MenteClara Vol. 1 (2) 2016. 


\section{Referencias}

Armagan N, Sayin HÜ, Kocatürk A (2012). Can Sexual Response be Enhanced and Expanded in the Human Female: Preliminary Findings and a Proposed Psychometric Scale for Expanded Sexual Response (ESR). 38th Annual Meeting of IASR (International Academy of Sex Research), Lisbon-Portual, July 8-11, 2012; Abtract Book, 38: 9.

Alzate H (1985). Vaginal eroticism: a replication study. Arch Sex Behav. 14: 529 -537.

Bancroft J (1989). Human sexuality and its problems. New York: Churchill Livingstone.

Bodansky S, Bodansky V (2000). Extended Massive Orgasm, California: Hunter House Pub.

Britten B (1983). The Love Muscle: Every Woman's Guide to Intensifying Sexual Pleasure, N.Y.: Signet Books.

Campbell B, \& Petersen WE (1953). Milk "let-down" and the orgasm in the human female. Human Biology, 25: 165-168.

Carellas B (2007). Urban Tantra. New York: Celestial Arts.

Chang J (1977). The Tao of Love and Sex: The Ancient Chinese Way to Ecstasy. New York: Dutton.

Chang J (1983). The Tao of the Loving Couple: True Liberation Through the Tao. New York: Dutton.

Chia M, Chia M, Abrams M, Abrams RC (2002). The Multi-Orgasmic Couple: Sexual Secrets Every Couple Should Know. New York: Harper One.

Chia M (2005). Healing Love through the Tao: Cultivating Female Sexual Energy. New York: Destiny Books.

Chua CA (1997). A proposal for a radical new sex therapy technique for the management of vasocongestion and orgasmic dysfunction in women: the AFE zone stimulation technique. Sex Marital Ther, 12:357-70.

Davidson JM, Davidson R J (1980). The psychobiology of consciousness. New York: Plenum Press.

Deadone Nicole (2011). Slow Sex: The Art and Craft of Female Orgasm, Boston: Grand Central-Life Style.

Ellis H (1897). Psychology of Sex. First printing: 1897, Fifth Printing: N.Y. Mentor Books.

Escapa Roy (1989). Bizzare Sex, London: Grafton Books.

Fisher S (1972). Female Orgasm, New York: Basic Books.

Fisher S (1973). Understanding the Female Orgasm, London: Penguin Books.

Freud S (1905). Three Essays on the Theory of Sexuality, 1905.

Glenn J \& Kaplan EH (1968). Types of orgasm in women: a critical review and redefinition. J Am Psychoanaly Ass, 16: 549 -564. 
Gómez, O. R. (2017). Tantra o Yoga. Estudios clínicos, $1^{a}$ parte: Yoga. Revista Científica Arbitrada de la Fundación MenteClara, 2(2), 63-84. DOI: https://doi.org/10.32351/rca.v2.2.11 https://fundacionmenteclara.org.ar/revista/index.php/RCA/article/view/11

Hite S (1976). The Hite Report: A National Study of Female Sexuality. New York: Dell Publishing.

Kaplan HS (1974). The New Sex Therapy, New York: Penguin Books.

King R, Belsky J, Mah K, Binik YM (2010). Are there different types of female orgasms? Arch Sex Behav, 40(5):865-75.

Kline-Graber G, Graber B (1975). A guide to sexual satisfaction: woman's orgasm. New York: Fawcett Popular Library.

Komisaruk BR, Beyer-Flores C, Whipple B (2006). The Science of Orgasm, Baltimore: John Hopkins University Press.

Ladas AK, Beverly W, Perry JD (1982-2005). The G-Spot and other Discoveries About Human Sexuality, New York: Henry Holtand Company.

Levin R (2014). The pharmacology of the human female orgasm - Its biological and physiological backgrounds. Pharmacol Biochem Behav, 121: 62-70.

Mah K, Binik YM (2001). The nature of orgasm: A critical review of major trends. Clin Psych Rev, 6:823-56.

Mah K, Binik YM (2005). Are orgasms in the mind or the body? Psychosocial versus physiological correlates of orgasmic pleasure and satisfaction. J Sex \& Marital Ther, 31:187-2005.

Masters W, Johnson V (1966). Human Sexual Response, Boston: Little Brown Company.

Michaels AM, Johnson P (2008). Tantra for Erotic Empowerment. Minnesota: Llewellyn Pub.

Morris D (2004). The Naked Woman: A Study of the Female Body. London: St. Martin's Griffin.

Muchembled R, Ferbach D (2008). Orgasm and the West: A History of Pleasure from the 16th Century to the Present, New York; Polity.

Mumford J (2005). Ecstasy through Tantra. Minnesota: Llewellyn Pub, 3rd ed.

Reich W (1973). The function of the orgasm: sex - economic problems of biological energy. New York: Farrar, Straus and Giroux (V.R. Carfagno, Translation; original work published 1942).

Reubens JR (1982). The physiology of normal sexual response in females. J Psychoactive Drugs, 14: $45-46$.

Rhodes R, Brauer A, Brauer DJ (1991). ESO Ecstasy Program: Better, Safer Sexual Intimacy, New York: Grand Central Publishing.

Ruck CAP (2006). Sacred Mushrooms of the Goddess: Secrets of Eleusis. Berkeley, CA: Ronin Publishing. 
Sayin HÜ (2010). Deep Sex: Different Dimensions and Openings of Sexuality (Derin Seks: Cinsellikte Farklı Boyutlar, Yeni Açılımlar), İstanbul: Klan Publications.

Sayin HÜ, Ramstadius M, Kocatürk A (2011-a). Expanded Desire: The Main Parameters and New Definitions of Enhanced and Expanded Sexual Response (ESR). The 33rd NACS Conference 2011, Oslo, Norway. Abstract Book, 33:21.

Sayin HÜ, Ramstadius M, Kocatürk A (2011-b). Pelvic Floor Muscle Strength is Correlated with Attaining Vaginal Orgasms in Human Female as Measured by Kegel Perineometer. The 33rd NACS Conference 2011, Oslo, Norway. Abstract Book, Abstract Book 2011; 33: 22.

Sayin HÜ (2011-c). Altered states of consciousness occurring during expanded sexual response in the human female: preliminary definitions. Neuroquantology; 9(4): 882-891.

Sayin HÜ, Can G, Ramstadius M, Kocatürk A, Kahya I (2011-d). Orgasmic Behavior Patterns in 2240 Educated Turkish Women Which Improves with Education. The 33rd NACS Conference 2011, Oslo, Norway. Abstract Book, Abstract Book 2011; 33: 22.

Sayin HÜ (2012-a). Expanded Sexual Response (ESR): Ultra Orgasm in Women (Artırılmış Cinsel Doyum: ESR). İstanbul: Tantra Akademi Publications.

Sayin HÜ, Kocatürk A (2012-b). Expanded Sexual Response in the Human Female: The Mechanisms of Expanded Orgasms in Women (Review). Proceedings of NACS-2012Helsinki Conference, Proceedings Book 2012; pp:147-163 (available at: http://www.expandedorgasms.net/pdf-sayin-etal/sayin-nacs-proceeding-paper-02.pdf

Sayin HÜ, Kocatürk A, Armagan N (2012-c). What Next After 60 Years of G-Spot Debate? Other Possible Erogenous Zones in Female Genitalia Which Can Contribute to the Development of Female Orgasm Other than Glans Clitoris. The 34th NACS-Sexology Conference (Pleasure and Health) 4-7 October, 2012, Helsinki-Finland. NACSConference Abstract Book 2012; 34: 30-31.

Sayin HÜ, Ramstadius M (2012-d). Altered States of Consciousness (ASC) During Female Orgasms and Expanded Sexual Response (ESR): Don't Use Drugs, Use Expanded Orgasms! The 34th NACS-Sexology Conference (Pleasure and Health) 4-7 October, 2012, Helsinki-Finland. NACS-Conference Abstract Book 2012; 34: 33.

Sayin HÜ, Kocatürk A, Kocatürk C, Ramstadius M (2012-e). Correlation of Factors that Contribute to the Masturbation Quality and the Strength of Pelvic Floor Muscles (PFM) as Measured by Kegel Perineometer. The 34th NACS-Sexology Conference (Pleasure and Health) 4-7 October, 2012, Helsinki-Finland. NACS-Conference Abstract Book 2012; 34: 32.

Sayin HÜ, Kece C (2012-f). Four Nerve and Six Pathway Theory of Female Orgasmic Response. The 34th NACS-Sexology Conference (Pleasure and Health) 4-7 October, 2012, Helsinki-Finland. NACS-Conference Abstract Book 2012; 34: 29.

Sayin HÜ, Kocatürk A, Kece C, Dastan K (2012-g). The Acute Effects of Nasal Oxytocin Spray on Female Behavior: Sedation, Arousal, Anxiolysis, Empathy and Altered States of Consciousness. The 34th NACS-Sexology Conference (Pleasure and Health) 4-7 October, 2012, Helsinki-Finland. NACS-Conference Abstract Book 2012; 34: 32. 
Sayin HÜ (2012-h). Doors of female orgasmic consciousness: New theories on the peak experience and mechanisms of female orgasm and expanded sexual response. (Research and Review) NeuroQuantology, 10 (4): 692-714.

Sayin HÜ \& Kocatürk A (2012-j). Expanded sexual response in the human female: The mechanisms of expanded orgasms in women (Review). Proceedings of NACS-2012Helsinki Conference, Helsinki-Finland (4-7 October, 2012) Proceedings Book 2012; pp: 147-163.

Sayin HÜ (2014-a). Other Dimensions of Sexuality (Cinsellikte Farklı Boyutlar, Derin Seks). Second edition, 2 volumes. İstanbul: Tantra Akademi publications.

Sayin HÜ (2014-b). The Consumption of Psychoactive Plants During Religious Rituals: The Roots of Common Symbols and Figures in Religions and Myths, (Research and Review) NeuroQuantology, 12 (2): 276-296.

Sayin HÜ (2015). Psychology of Female Orgasm (Kadınlarda Orgazmin Psikolojisi). İstanbul: Tantra Akademi publications.

Sayin HÜ (2016). Sexual Disorders and Paraphilia (Cinsel Bozukluklar ve Parafili). Textbook. İstanbul: Tantra Akademi.

Schwartz L, Schwartz B (1999). The One Hour Orgasm. New York: St. Martin's Griffin.

Schiavi R C, Segraves R T (1995). The biology of sexual function. Psychiat Clin North Am, 18: 7-23.

Sukel K (2011). Sex on the brain: Orgasms unlock altered consciousness. New Scientist 2011; 2812. http://www.newscientist.com/article/mg21028124.600-sex-on-the-brainorgasms-unlock-altered-consciousness.html?full=true .

Tannahill R (1992). Sex in History, New York: Scharborough House.

Taylor P (2000). PhD Thesis: An Observational and Comparative Study of Practitioners of Expanded Orgasm: An Investigation of an Effective and Accessible Path to Transcendent States of Consciousness, Submitted in Partial Fulfillment of the Requirements for the Degree of Philosophy in Transpersonal Psychology, International University of Professional Studies, Maui, Hawaii.

Taylor P (2002). Expanded Orgasm: Soar to Ecstasy at your Lover's Every Touch, Illinois: Sourcebooks, Casablanca.

Taylor (2004). DVD Video, Expand Her Orgasm Tonight.

Vatsyayana, Kama Sutra, Trans. by Burton R., London: Private, 1883.

Wu W and Humana C (1996). Chinese Sex Secrets, New York: CFV Publications.

Zdrok V (2004). Anatomy of Pleasure. Philadelphia: Infinity Publishing Co. 